\title{
Removal of poly- and perfluorinated compounds from ion exchange regenerant still bottom samples in a plasma reactor
}

Raj Kamal Singh ${ }^{1}$, Nicholas Multari ${ }^{1}$, Chase Nau-Hix ${ }^{1}$, Steven Woodard ${ }^{2}$, Michael Nickelson ${ }^{2}$, Selma Mededovic Thagard ${ }^{1}$, Thomas M. Holsen*3

${ }^{1}$ Plasma Research Laboratory, Department of Chemical and Biomolecular Engineering, Clarkson University, Potsdam, New York, United States

${ }^{2}$ Emerging Compounds Treatment Technologies $\left(\mathrm{ECT}_{2}\right)$ Inc., Portland, ME, USA

${ }^{3}$ Department of Civil and Environmental Engineering, Clarkson University, Potsdam, New York, United States

* Corresponding author; E-mail: tholsen@clarkson.edu; \#+1-315-268-3851

This supplementary information file contains 6 Tables and 3 Texts. 
Table S1. Gradient solvent program for the UPLC

\begin{tabular}{|c|c|c|c|}
\hline Time $(\mathrm{min})$ & $\begin{array}{c}\text { Flowrate } \\
(\mathrm{mL} / \mathrm{min})\end{array}$ & $\% \mathrm{~A}$ & $\% \mathrm{~B}$ \\
\hline 0.0 & 0.5 & 70 & 30 \\
\hline 0.5 & 0.5 & 70 & 30 \\
\hline 3.0 & 0.5 & 10 & 90 \\
\hline 3.1 & 0.5 & 0 & 100 \\
\hline 4.5 & 0.5 & 0 & 100 \\
\hline 4.6 & 0.5 & 70 & 30 \\
\hline 6.5 & 0.5 & 70 & 30 \\
\hline
\end{tabular}

Table S2. List of calibration standards, surrogate standards and minimum detection limit (MDL) used for perfluorinated alkyl acids (PFAAs)

\begin{tabular}{|c|c|c|c|c|}
\hline $\begin{array}{c}\text { Analyte } \\
\text { abbreviation }\end{array}$ & Analyte name & $\begin{array}{l}\text { Surrogate } \\
\text { standard }\end{array}$ & Surrogate name & $\begin{array}{l}\text { MDL } \\
(\mathrm{ng} / \mathrm{L})\end{array}$ \\
\hline PFBA & $\begin{array}{l}\text { Perfluorobutanoic } \\
\text { acid }\end{array}$ & {$\left[{ }^{13} \mathrm{C}_{4}\right] \mathrm{PFBA}$} & $\begin{array}{l}\text { perfluoro-n- }\left[{ }^{13} \mathrm{C}_{4}\right] \\
\text { butanoic acid }\end{array}$ & 25 \\
\hline PFPeA & $\begin{array}{l}\text { Perfluoropentanoic } \\
\text { acid }\end{array}$ & {$\left[{ }^{13} \mathrm{C}_{2}\right] \mathrm{PFHxA}$} & $\begin{array}{l}\text { perfluoro-n- }\left[1,2-{ }^{13} \mathrm{C}_{2}\right] \\
\text { hexanoic acid }\end{array}$ & 10 \\
\hline PFBS & $\begin{array}{l}\text { Perfluorobutane } \\
\text { sulfonate }\end{array}$ & {$\left[{ }^{18} \mathrm{O}_{2}\right] \mathrm{PFHxS}$} & $\begin{array}{l}\text { perfluoro-n-hexane }\left[{ }^{18} \mathrm{O}_{2}\right] \\
\text { sulfonate }\end{array}$ & 15 \\
\hline PFHxA & $\begin{array}{l}\text { Perfluorohexanoic } \\
\text { acid }\end{array}$ & {$\left[{ }^{13} \mathrm{C}_{2}\right] \mathrm{PFHxA}$} & $\begin{array}{c}\text { perfluoro-n- }\left[1,2-{ }^{13} \mathrm{C}_{2}\right] \\
\text { hexanoic acid }\end{array}$ & 20 \\
\hline PFPeS & $\begin{array}{c}\text { Perfluoropentane } \\
\text { sulfonate }\end{array}$ & {$\left[{ }^{18} \mathrm{O}_{2}\right] \mathrm{PFHxS}$} & $\begin{array}{l}\text { perfluoro-n-hexane }\left[{ }^{18} \mathrm{O}_{2}\right] \\
\text { sulfonate }\end{array}$ & 15 \\
\hline PFHpA & $\begin{array}{l}\text { Perfluoroheptanoic } \\
\text { acid }\end{array}$ & {$\left[{ }^{13} \mathrm{C}_{4}\right] \mathrm{PFOA}$} & $\begin{array}{l}\text { perfluoro-n- }[1,2,3,4- \\
\left.{ }^{13} \mathrm{C}_{4}\right] \text { octanoic acid }\end{array}$ & 20 \\
\hline PFHxS & $\begin{array}{c}\text { Perflorohe xane } \\
\text { sulfonate }\end{array}$ & {$\left[{ }^{18} \mathrm{O}_{2}\right] \mathrm{PFHxS}$} & $\begin{array}{l}\text { perfluoro-n-hexane }\left[{ }^{18} \mathrm{O}_{2}\right] \\
\text { sulfonate }\end{array}$ & 20 \\
\hline PFOA & Perfluorooctanoic acid & {$\left[{ }^{13} \mathrm{C}_{4}\right] \mathrm{PFOA}$} & $\begin{array}{l}\text { perfluoro-n-[1,2,3,4- } \\
\left.{ }^{13} \mathrm{C}_{4}\right] \text { octanoic acid }\end{array}$ & 15 \\
\hline PFHpS & $\begin{array}{l}\text { Perfluoroheptane } \\
\text { sulfonate }\end{array}$ & {$\left[{ }^{13} \mathrm{C}_{4}\right] \mathrm{PFOS}$} & $\begin{array}{c}\text { perfluoro-n- }[1,2,3,4- \\
\left.{ }^{13} \mathrm{C}_{4}\right] \text { octane sulfonates }\end{array}$ & 35 \\
\hline PFNA & Perfluoronanoic acid & {$\left[{ }^{13} \mathrm{C}_{5}\right] \mathrm{PFNA}$} & $\begin{array}{l}\text { perfluoro-n- }[1,2,3,4,5- \\
\left.{ }^{13} \mathrm{C}_{5}\right] \text { nonanoic acid } \\
\end{array}$ & 15 \\
\hline PFOS & $\begin{array}{l}\text { Perfluorooctane } \\
\text { sulfonate }\end{array}$ & {$\left[{ }^{13} \mathrm{C}_{4}\right] \mathrm{PFOS}$} & $\begin{array}{c}\text { perfluoro-n-[1,2,3,4- } \\
\left.{ }^{13} \mathrm{C}_{4}\right] \text { octane sulfonates }\end{array}$ & 35 \\
\hline PFDA & $\begin{array}{l}\text { Perfluorodecanoic } \\
\text { acid }\end{array}$ & {$\left[{ }^{13} \mathrm{C}_{2}\right] \mathrm{PFDA}$} & $\begin{array}{c}\text { perfluoro-n- }\left[1,2-{ }^{13} \mathrm{C}_{2}\right] \\
\text { decanoic acid }\end{array}$ & 15 \\
\hline PFNS & $\begin{array}{c}\text { Perfluoronanone } \\
\text { sulfonate }\end{array}$ & {$\left[{ }^{13} \mathrm{C}_{4}\right] \mathrm{PFOS}$} & $\begin{array}{c}\text { perfluoro-n- }[1,2,3,4- \\
\left.{ }^{13} \mathrm{C}_{4}\right] \text { octane sulfonates } \\
\end{array}$ & 40 \\
\hline PFDS & $\begin{array}{l}\text { Perfluorodecane } \\
\text { sulfonate }\end{array}$ & {$\left[{ }^{13} \mathrm{C}_{2}\right] \mathrm{PFOS}$} & $\begin{array}{l}\text { perfluoro-n-[1,2,3,4- } \\
\left.{ }^{13} \mathrm{C}_{4}\right] \text { octane sulfonates }\end{array}$ & 45 \\
\hline
\end{tabular}




\begin{tabular}{|c|c|c|c|c|}
\hline PFUnA & $\begin{array}{c}\text { Perfluoroundecanoic } \\
\text { acid }\end{array}$ & {$\left[{ }^{13} \mathrm{C}_{2}\right] \mathrm{PFUnA}$} & $\begin{array}{c}\text { perfluoro-n- }\left[1,2-{ }^{13} \mathrm{C}_{2}\right] \\
\text { undecanoic acids }\end{array}$ & 80 \\
\hline PFDoA & $\begin{array}{c}\text { Perfluorododecanoic } \\
\text { acid }\end{array}$ & {$\left[{ }^{13} \mathrm{C}_{2}\right] \mathrm{PFDoA}$} & $\begin{array}{c}\text { perfluoro-n- }\left[1,2-{ }^{13} \mathrm{C}_{2}\right] \\
\text { dodecanoic acid }\end{array}$ & 25 \\
\hline PFTrA & $\begin{array}{c}\text { Perfluorotridecanoic } \\
\text { acid }\end{array}$ & {$\left[{ }^{13} \mathrm{C}_{2}\right] \mathrm{PFDoA}$} & $\begin{array}{c}\text { perfluoro-n- }\left[1,2-{ }^{13} \mathrm{C}_{2}\right] \\
\text { dodecanoic acid }\end{array}$ & 20 \\
\hline PFTeA & $\begin{array}{c}\text { Perfluorotetradecanoic } \\
\text { acid }\end{array}$ & {$\left[{ }^{13} \mathrm{C}_{2}\right] \mathrm{PFTeA}$} & $\begin{array}{c}\text { perfluoro-n- }\left[1,2-{ }^{13} \mathrm{C}_{2}\right] \\
\text { tetradecanoic acid }\end{array}$ & 35 \\
\hline
\end{tabular}

Note: $M D L$ are reported in 4 times diluted samples

Table S3. List of calibration standards used for PFAAs precursors, surrogate standards and MDL.

\begin{tabular}{|c|c|c|c|c|}
\hline $\begin{array}{c}\text { Analyte } \\
\text { abbreviation }\end{array}$ & Analyte name & $\begin{array}{c}\text { Surrogate } \\
\text { standard }\end{array}$ & Surrogate name & $\begin{array}{l}\text { MDL } \\
(\mathrm{ng} / \mathrm{L})\end{array}$ \\
\hline 10:2 FTS & $\begin{array}{l}\text { 10:2 Fluorotelomere } \\
\text { sulfonate }\end{array}$ & d-N-MeFOSA-M & $\begin{array}{l}\text { N-methyl-d3-perfluoro-1- } \\
\text { octanesulfonamide }\end{array}$ & 25 \\
\hline 4:2 FTS & $\begin{array}{l}\text { 4:2 Fluorotelomere } \\
\text { sulfonate }\end{array}$ & d-N-MeFOSA-M & $\begin{array}{l}\text { N-methyl-d3-perfluoro-1- } \\
\text { octanesulfonamide }\end{array}$ & 15 \\
\hline $6: 2$ FTS & $\begin{array}{c}\text { 6:2 Fluorotelomere } \\
\text { sulfonate }\end{array}$ & d-N-MeFOSA-M & $\begin{array}{l}\text { N-methyl-d3-perfluoro-1- } \\
\text { octanesulfonamide }\end{array}$ & 35 \\
\hline 8:2 FTS & $\begin{array}{c}8: 2 \text { Fluorotelomere } \\
\text { sulfonate }\end{array}$ & d-N-MeFOSA-M & $\begin{array}{c}\text { N-methyl-d3-perfluoro-1- } \\
\text { octanesulfonamide }\end{array}$ & 30 \\
\hline EtFOSA & $\begin{array}{l}\text { N-Ethyl perfluorooctane } \\
\text { sulfonamide }\end{array}$ & d-N-EtFOSA-M & $\begin{array}{l}\text { N-ethyl-d3-perfluoro-1- } \\
\text { octanesulfonamide }\end{array}$ & 25 \\
\hline EtFOSAA & $\begin{array}{l}\text { N-Ethyl perfluorooctane } \\
\text { sulfonamide acetic acid }\end{array}$ & d3-N-EtFOSAA & $\begin{array}{l}\text { N-ethyl-d3-perfluoro-1- } \\
\text { octanesulfonamidoacetic } \\
\text { acid }\end{array}$ & 65 \\
\hline FOSA-1 & $\begin{array}{c}\text { Perfluoro-1-octane } \\
\text { sulfonamide }\end{array}$ & d-N-MeFOSA-M & $\begin{array}{c}\text { N-methyl-d3-perfluoro-1- } \\
\text { octanesulfonamide }\end{array}$ & 20 \\
\hline FOSAA & $\begin{array}{c}\text { Perfluorooctane } \\
\text { sulfonamide acetic acid }\end{array}$ & d-N-MeFOSA-M & $\begin{array}{l}\text { N-methyl-d3-perfluoro-1- } \\
\text { octanesulfonamide }\end{array}$ & 30 \\
\hline MeFOSA & $\begin{array}{c}\text { N-Methyl } \\
\text { perfluorooctane } \\
\text { sulfonamide }\end{array}$ & d-N-MeFOSA-M & $\begin{array}{l}\text { N-methyl-d3-perfluoro-1- } \\
\text { octanesulfonamide }\end{array}$ & 35 \\
\hline MeFOSAA & $\begin{array}{c}\text { N-Methyl } \\
\text { perfluorooctane } \\
\text { sulfonamide acetic acid }\end{array}$ & d3-N-MeFOSAA & $\begin{array}{c}\text { N-methyl-d3-perfluoro-1- } \\
\text { octanesulfonamidoacetic } \\
\text { acid }\end{array}$ & 55 \\
\hline
\end{tabular}

Note: $M D L$ are reported in 4 times diluted samples 
Table S4. Total oxidizable precursor (TOP) concentrations reduction in six different IX brine regenerant still bottom samples. TOP concentrations are normalized to initial (without dilution) in SB samples. $\mathrm{HC1A}^{*}$ was undiluted, $\mathrm{HC1C}^{* *}$ was diluted 50 times, and other SB samples were diluted 10 times.

\begin{tabular}{|l|l|l|l|}
\hline & $\begin{array}{l}\text { TOP Initial } \\
(\mathrm{mg} / \mathrm{L})\end{array}$ & $\begin{array}{l}\text { TOP final } \\
(\mathrm{mg} / \mathrm{L})\end{array}$ & $\begin{array}{l}\text { TOP removal } \\
(\%)\end{array}$ \\
\hline HC1A $^{*}$ & 3870 & 2 & 99.9 \\
\hline HC1B & 9260 & 34 & 99.6 \\
\hline HC1C** & 6870 & 13 & 99.8 \\
\hline A3FA & 4380 & 10 & 99.9 \\
\hline A3FB & 6360 & 11 & 99.8 \\
\hline A3FC & 9440 & 15 & 99.8 \\
\hline
\end{tabular}

Table S5. Fluoride production in six different IX brine regenerant still bottom samples. Concentrations are normalized to initial (without dilution) in SB samples. HC1A* was undiluted, $\mathrm{HC1C}^{* *}$ was diluted 50 times, and other SB samples were diluted 10 times.

\begin{tabular}{|l|l|l|}
\hline & $\begin{array}{l}\text { Initial } \\
(\mathrm{mg} / \mathrm{L})\end{array}$ & $\begin{array}{l}\text { Final } \\
(\mathrm{mg} / \mathrm{L})\end{array}$ \\
\hline HC1A $^{*}$ & 340 & 7890 \\
\hline HC1B & 1670 & 8420 \\
\hline HC1C** & 4700 & 16800 \\
\hline A3FA & 1600 & 10700 \\
\hline A3FB & 360 & 14400 \\
\hline A3FC & 570 & 8800 \\
\hline
\end{tabular}


Table S6. Chlorite $\left(\mathrm{ClO}_{2}^{-}\right)$and chlorate $\left(\mathrm{ClO}_{3}^{-}\right)$production in six different IX brine regenerant still bottom samples. Concentrations are normalized to initial concentration (without dilution) in SB samples. $\mathrm{HC1A}^{*}$ was undiluted, $\mathrm{HC1C}^{* * *}$ was diluted 50 times, and other SB samples were diluted 10 times.

\begin{tabular}{|l|l|l|l|l|}
\hline \multirow{2}{*}{ SBs } & \multicolumn{2}{|c|}{ Chlorite (mM) } & \multicolumn{2}{c|}{ Chlorate (mM) } \\
\cline { 2 - 5 } & Untreated & Treated & Untreated & Treated \\
\hline HC1A $^{*}$ & 1 & 10 & 1 & 6 \\
\hline HC1B & BDL & 15 & 1 & 132 \\
\hline HC1C** & 37 & 36 & 64 & 720 \\
\hline A3FA & BDL & 23 & 1 & 76 \\
\hline A3FB & 35 & 146 & 27 & 100 \\
\hline A3FC & BDL & 13 & 3 & 97 \\
\hline
\end{tabular}

\section{Text S1 - Resin regeneration process}

The regeneration procedure was as follows:

Step 1 - The column was flushed with $1 \mathrm{BV}(1.98 \mathrm{~L})$ of a $2 \% \mathrm{w} / \mathrm{w} \mathrm{NaCl}$ solution at $2 \mathrm{BV} / \mathrm{hr}$ (66 $\mathrm{mL} / \mathrm{min}$ ) for 30 minutes. The rinsate was collected into a $4 \mathrm{~L}$ container.

Step 2 - The column was regenerated with a $10 \mathrm{BV}(19.8 \mathrm{~L})$ of a $70 \% \mathrm{v} / \mathrm{v}$ alcohol $/ 2 \% \mathrm{w} / \mathrm{w} \mathrm{NaCl}$ solution $2 \mathrm{BV} / \mathrm{hr}(66 \mathrm{~mL} / \mathrm{min})$ for 360 minutes. The spent regenerant was collected in two $20 \mathrm{~L}$ carboys.

Step 3 - The column was flushed with $2 \mathrm{BV}(3.96 \mathrm{~L})$ of distilled water $4 \mathrm{BV} / \mathrm{hr}(132 \mathrm{~mL} / \mathrm{min})$ for 30 minutes. The rinsate was collected into a $4 \mathrm{~L}$ container.

Step 4 - The column was flushed with $4 \mathrm{BV}(7.92 \mathrm{~L})$ of distilled water $4 \mathrm{BV} / \mathrm{hr}(132 \mathrm{~mL} / \mathrm{min})$ for 60 minutes. The rinsate was collected into a $20 \mathrm{~L}$ container.

Still bottoms were obtained by distilling 3 liters of spent regenerant from the first $20 \mathrm{~L}$ carboy from Step 2. 


\section{Text S2 - Analytical procedure for PFAS analysis}

\section{Methods}

A UPLC-MS-MS (Thermo Scientific, Vanquish-TSQ ALTIS) was used for the analysis of PFAS and their precursors. Separation was performed with a Phenomenex Luna Omega PS C18 column $(2.1 \mathrm{~mm} \times 100 \mathrm{~mm}, 1.6 \mu \mathrm{m})$ and samples were analyzed by electrospray in negative ion mode. Samples were diluted with methanol (1:3 ratio) and then sonicated and centrifuged prior to injection $(10 \mu \mathrm{L})$. A gradient solvent program was operated with $5 \mathrm{mM}$ ammonium acetate in LCMS grade water (solution A) and a LCMS grade acetonitrile (solution B). The details of the gradient method are provided in Table S1. Mass range was set at 50 to $950 \mathrm{Da}$ with a scan rate of $0.055 / \mathrm{sec}$.

\section{Quality Assurance}

For quality assurance and control, all samples were spiked with 2 ng of labeled internal standards. Based on the analysis of method blanks, the limit of detection (LOD) was determined to be $3 \mathrm{ng} / \mathrm{L}$. Six point calibration in the range of 9 and $20000 \mathrm{ng} / \mathrm{L}$ was used for the quantification of samples using internal standard methods (details are provided in Table S2 and S3). Quantification was carried out using TraceFinder software from Thermo Scientific using regression fit of $r^{2}>0.99$ and deviation <30\%. Calibration standards were reinjected in the sample sequence to validate the time-dependent response from the instruments. Analyzed data were quantified if surrogate recovery was between 70 and $120 \%$. 


\section{Text S3. Total oxidizable precursors (TOP) analysis}

An earlier method developed by Houtz and Sedlak ${ }^{1}$ demonstrated that an excess of hydroxyl radicals $\left({ }^{\circ} \mathrm{OH}\right)$ generated in persulfate oxidation fully converted PFAS precursors to PFAS. Samples were diluted to 10000 times prior to heating samples at $85^{\circ} \mathrm{C}$ in the oven. To evaluate this method, a mixture of common PFAS precursors containing different carbon chain-lengths including 10:2 FTS, 4:2 FTS, 6:2 FTS, 8:2 FTS, EtFOSA, EtFOSAA, FOSA-1, FOSAA, MeFOSA and MeFOSAA were used. The control sample did not contain methanol or other organic solvents. The dilution was made in deionized water from the mixture of precursor's stock solution of 50 $\mu \mathrm{g} / \mathrm{L}$. The prepared solution containing the mixture of precursors was transferred in $50 \mathrm{~mL}$ high density polyethylene (HDPE) tubes with a headspace $<1 \mathrm{~mL}$. Next, $60 \mathrm{mM}$ potassium persulfate and $10 \mathrm{~N} \mathrm{NaOH}$ solution were added to the precursors solution to maintain the $\mathrm{pH}$ above 12 . Experiments were performed in duplicate, with an aliquot used as a room temperature control (i.e., it was kept at room temperature after addition of persulfate and $\mathrm{NaOH}$ solutions). The HDPE tubes were placed in a temperature-controlled oven at temperature $92^{\circ} \mathrm{C}$ for $6 \mathrm{~h}$, and samples were analyzed after cooling at room temperature. A good conversion of all precursors to PFAAs (96.7 $\pm 4.3 \%$ ) was achieved.

\section{Reference}

1. Houtz, E. F.; Sedlak, D. L. Oxidative conversion as a means of detecting precursors to perfluoroalkyl acids in urban runoff. Environ. Sci. Technol. 2012, 46, 9342-9349. 